\title{
Analysis of optical properties and mechanism of photoluminescence enhancement of a quantum dot - ferroelectric liquid crystal composite
}

\author{
Tripti Vimal, ${ }^{1}$ Dharmendra P. Singh, ${ }^{1,2}$ Kaushlendra Agrahari, ${ }^{1}$ Atul Srivastava, ${ }^{1}$ and Rajiv Manohar ${ }^{* 1}$ \\ ${ }^{1}$ Liquid Crystal Research Lab, Physics Department, University of Lucknow, Lucknow, India-226007, \\ ${ }^{2}$ Unité de Dynamique et Structure des Matériaux Moléculaires, EA 4476, Université du Littoral Côte d'Opale, \\ F-59140 Dunkerque, France
}

Received November 09, 2016; accepted January 13, 2016; published March 31, 2016

\begin{abstract}
ZnS QDs (Quantum Dots) were dispersed in an FLC mixture (SCE4). The dispersion of QDs in FLC causes enhanced Photoluminescence, which can be attributed to the high molecular orderning of the composite system induced by QDs. FWHM (full width half maxima) values of peaks reveal that the presence of QDs in an FLC material faours the NB (near band) emissions. The POM (Polarizing Optical Microscope) images confirms the change in the alignment of FLC molecules after the dispersion of QDs.
\end{abstract}

The combination of LCs with nanomaterials has been studied widely in the recent years. The amalgamation of nanomaterials in Liquid Crystals (LCs) is a novel method to improve the various properties of LCs [1-3]. The ferroelectric liquid crystal (FLC), usually referred to as a chiral smectic $\mathrm{C}\left(\mathrm{SmC}^{*}\right)$ phase, has characteristic features like fast response time, low threshold voltage, good optical contrast, etc [4-5]. In the recent years more and more studies focused on the optical properties of FLCs have been carried out. In the present paper, a $\mathrm{ZnS}$ quantum dot has been dispersed in an FLC material (SCE4). The changes in the PL and optical properties after the dispersion of QDs have been analyzed. We have observed an almost threefold enhancement in the PL intensity for the QDs - FLC composite. The Gaussian fit of the PL peak of FLC and QDs - FLC composites has been done to obtain the components of the PL peak. The FWHM (full width half maxima) of peaks were found to be decreased, which indicates that the presence of QDs are favouring the NB (near band) emissions in the composites.

The sample cells used in the present study were prepared by using indium tin oxide (ITO) coated glass plates. The thickness of the sample cells was maintained $5 \mu \mathrm{m}$ by using Mylar spacers. $\mathrm{ZnS}$ is a typical II - VI semiconductor, it is widely used as a phosphor material and in thin film electroluminescent devices [6]. The phase sequence of the FLC material (SCE4) used here is as follows:

\footnotetext{
*E-mail: rajiv.manohar@gmail.com
}

Cryst., SmC*, SmA, N*, Iso. at $10^{\circ} \mathrm{C}, 57^{\circ} \mathrm{C}, 88^{\circ} \mathrm{C}, 122^{\circ} \mathrm{C}$ The $\mathrm{ZnS}$ QDs - FLC composites were prepared by dispersing an appropriate amount of $\mathrm{ZnS}$ QDs \{Mix.1 (0.25 wt. /wt. \%) and Mix.2 (0.5 wt. /wt. \%) \}into a pure

FLC material and homogenized by an ultrasonic mixer for $1 \mathrm{hr}$ at $122^{\circ} \mathrm{C}$. The PL spectra of all the samples were recorded using a Perkin Elmer LS55 luminescence spectrophotometer equipped with a Xenon discharge lamp of power equivalent to $20 \mathrm{~kW}$ for $8 \mathrm{~ms}$ duration, as an excitation source with slit width ( $5 \mathrm{~nm}$ in our case) at room temperature.

The optical micrographs of a pure FLC and QDs - FLC composite were taken using a polarizing microscope under crossed polarizer conditions in a planar aligned cell as shown in Figs. 1(a), (b) and (c). It is clearly observable from the POM images of the pure and QD - FLC composite that the surface morphology or the alignment of the pure FLC has been significantly changed after the dispersion of QDs. The change in the alignment of a pure FLC material with the addition of QDs is mainly due to the molecular alignment ability of QDs [7]. It is expected that the change in the molecular alignment also affects the optical property (like Photoluminescence) of FLC as phtoluminescence of any material depends upon the surface properties of that material. In the present study, the presence of QDs significantly changes the surface property (molecular alignment) and leads to subsequent changes in the PL properties of QDs - FLC composites.

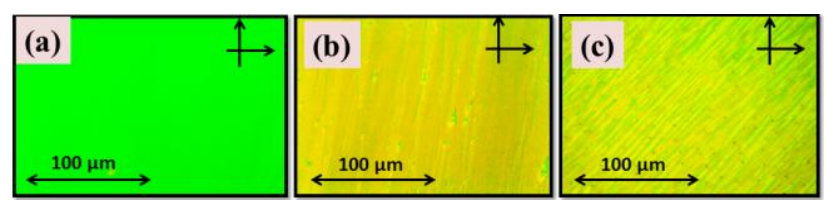

Fig. 1. Polarizing optical micrographs (POMs) of planar aligned samples of (a) pure (b) Mix. 1 and (c) Mix. 2 taken at $35^{\circ} \mathrm{C}$ under crossed polarizer conditions. 
The PL emission of a $\mathrm{ZnS}$ quantum dot has been represented in Fig. 2. Quantum dots emit strongly with a maximum at $420 \mathrm{~nm}$ with an excitation wavelength of $350 \mathrm{~nm}$. The blue emission centered at $420 \mathrm{~nm}$ is associated with the luminescence from the self-activated centers. These centers have often been attributed to crystal lattice vacancies [8].

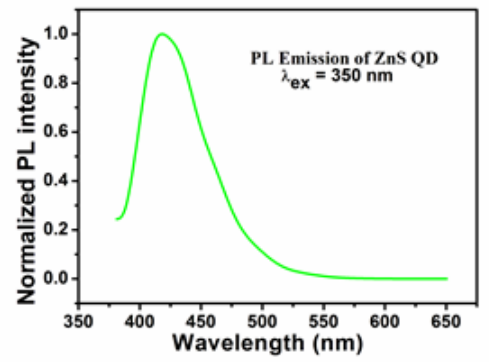

Fig. 2. PL emission spectra of $\mathrm{ZnS}$ quantum dot.

The PL emission plot of the FLC material and QDs FLC composite at a $350 \mathrm{~nm}$ excitation wavelength is depicted in Fig. 3(a). It can be seen that the FLC material emits strongly with a maximum at $\sim 394 \mathrm{~nm}$. Emission spectra are broad in nature and composed of three submerged components, as presented in Fig. 3 (b).
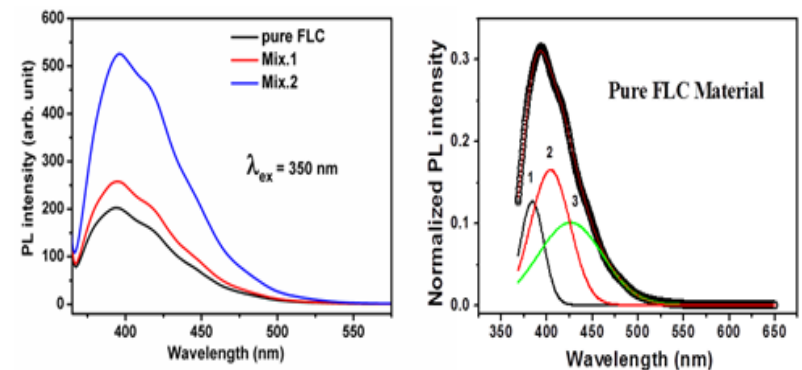

Fig. 3. (a) Photoluminescence spectra of FLC material and QDs - FLC composites. (b) The de-convoluted PL spectra of FLC material.

The de-convoluted spectra obtained from the Gaussian fit for the PL peak of the FLC material have three submerged components centered at 384, 404, 425nm. SCE4 FLC material is a mixture of molecules with different substituents. The three emission peaks of the FLC material show that different components of ferroelectric liquid crystal are contributing to the photoluminescence property of the ferroelectric mix. The wavelength band from 380-450nm corresponds to the violet region of visible light electromagnetic spectra. Three different emission peaks predict that all the three http://www.photonics.pl/PLP have the same origin with a slight change in the molecular structure of the PL sources [9].

PL emission spectra of QDs - FLC composite depict a substantial increase in the intensity of PL emission of an FLC material after the dispersion of QDs. It seems that the emission bands of the FLC material have been modified and shifted by the dispersion of the $\mathrm{ZnS}$ quantum dot. The PL emission for Mix.1 and for Mix.2 was found to be centred at $395 \mathrm{~nm}$ and $396 \mathrm{~nm}$, respectively. The deconvoluted PL spectra for both the composites are shown in Fig. 4(b) and Fig. 4(c).
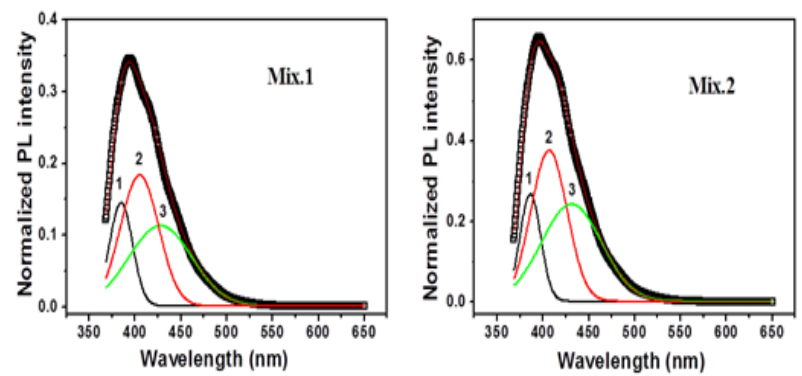

Fig. 4. (a)-(b) De-convoluted PL spectra of Mix.1 and Mix.2.

The fitted PL peak spectra for mixtures again result in three emission peaks. For Mix.1, the position of peak has been found to be at $384,405,427 \mathrm{~nm}$, respectively. However, for Mix.2 the peaks are centered at 386, 406, $432 \mathrm{~nm}$, respectively. The PL intensity for the QD dispersed FLC has been enhanced by almost threefold for Mix.2. Such a large enhancement can be attributed to high molecular ordering due to the presence of a quantum dot. It means that a high dipole moment of QDs [10] leads to additional orderning of an FLC molecule, which subsequently affects the surface morphology of the composite system as discussed earlier in the manuscript. So it can be concluded that the emission from the QDs is combining constructively with the emission from the highly scattering phase of the FLC material due to the high orderning of an FLC molecule in the composite system [11]. We found no emission peak related to the pristine QDs in the PL spectra of the $\mathrm{ZnS}$ - FLC composite system. This indicates that the QDs based FLC composite shows different PL properties rather than individual constituents. The intensity of emission peaks strongly depends on dopant concentration. A higher concentration of QDs leads to a higher ordering in the system, consequently higher PL intensity [12]. 


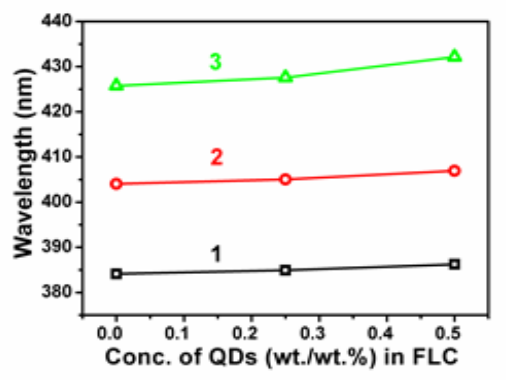

Fig. 5. Variation in the wavelength of the three components of a PL peak with a variation in the conc. of QDs (wt./wt. \%) in a host FLC material. 1, 2, 3 refer to PL peak components as depicted in the deconvoluted spectra of an FLC material and QDs - FLC composites.

It has been observed that the position of first and second emission peaks has been shifted towards a higher wavelength by about 1 or $2 \mathrm{~nm}$ for QDs - FLC composites. However, the position of a third emission peak has been red shifted by about $8 \mathrm{~nm}$ for Mix.2. This indicates that the concentrations of QDs plays an important role in modifying the properties of the host. The FWHM (Full Width Half Maximum) value of all the emission peak has been presented in the following Table.

\begin{tabular}{|c|c|c|c|}
\hline $\begin{array}{c}\text { FWHM (nm) } \\
\text { of the peaks } \rightarrow\end{array}$ & Peak 1 & Peak 2 & Peak 3 \\
\hline Pure FLC & 25 & 43 & 71 \\
\hline Mix.1 & 25 & 41 & 69 \\
\hline Mix.2 & 22 & 38 & 65 \\
\hline
\end{tabular}

It can be seen that the FWHM value of all the emission peaks has been decreased for QDs - FLC composites. The emission peak having FWHM $<25 \mathrm{~nm}$ corresponds to the NB (near band) Emission and FWHM $>25 \mathrm{~nm}$ is mainly due to surface defect level transitions [13]. It has been reported by Lupon et al. and Bae et al., that FWHM < $25 \mathrm{~nm}$ is mainly due to direct transitions of electrons between the conduction band and valence band tails [1415]. However, defect levels are shallow levels with respect to the band edges. The small decrease in the FWHM value for QDs - FLC composites predicts that in the present study QDs are supporting radiative emissions from bands rather than defects levels of an FLC material.

In brevity, it can can be concluded that the dispersion of ZnS QDs into the host FLC material changes its optical parameters. A change in the aligment of FLC molecules in composites has been reflected in their PL profile. The PL intensity has been enhanced significantly and it has been found that the QDs favour the NB (near band) emissions in the composites. Thus the present study provides a better understanding of the optical properties of QDs - FLC composites for the development of display technology.
One of the authors is thankful to DST for the grant in the form of an INDO-POLISH project. Author TV is sincerely thankful to UGC, New Delhi for providing financial assistance in the form of the UGC-BSR Fellowship. DPS is also thankful to CEFIPRA for the Raman-Charpak fellowship in France. RM is thankful to UGC for the grant of a research award (2014-16).

\section{References}

[1] S.W. Lee, C. Mao, C.E. Flynn, A.M. Belcher, Science 3, 892 (2002).

[2] M. Tamborra, M. Striccoli, R. Comparelli, M.L. Curri, A. Petrella, A. Agostiano, Nanotechn. 15(4), S240 (2004).

[3] E. Kikuchi, S. Kitada, A. Ohno, S. Aramaki, S. Maenosono, Appl. Phys. Lett. 92, 173307 (2008).

[4] T. Joshi, A. Kumar, J. Prakash, A.M. Biradar, Appl. Phys. Lett. 96, 253109 (2010).

[5] S. K. Gupta, D.P. Singh, P.K. Tripathi, R. Manohar, M. Varia, L.K. Sagar, S. Kumar, Liq. Cryst. 40, 528 (2013).

[6] A.K. Kole, C.S. Tiwary, P. Kumbhakar, J. Appl. Phys. 113, 114308 (2013).

[7] H. Qi, T. Hegmann, ACS Appl. Mater. Interfaces 1, 1731 (2009).

[8] E. Mohagheghpour, M. Rabiee, F. Moztarzadeh, M. Tahriri, J. Ceramic Proc. Research 11(2), 144 (2010).

[9] P. Goel, M. Arora, RSC Adv. 5, 14974 (2015).

[10] I.A. Ostapenko, G. Honig, C. Kindel, S. Rodt, A.Strittmatter, A. Hoffmann, D. Bimberg, Appl. Phys. Lett. 97, 063103 (2010).

[11] A. Kumar , J. Prakash, A.D. Deshmukh , D. Haranath, P. Silotia, A.M. Biradar, Appl. Phys. Lett. 100, 134101 (2012).

[12] A. Kumar, S. Tripathi, A.D. Deshmukh, D. Haranath, P. Singh, A.M. Biradar, J. Phys. D: Appl. Phys. 46, 195302 (2013).

[13] D.P. Singh, S.K. Gupta, A. Srivastava, R. Manohar, J. Luminescence 139, 60 (2013).

[14] O. Lupan, S. Shishiyanu, V. Ursaki, H. Khallaf, L. Chow et al., Solar Energy Materials \& Solar Cells 93, 1417 (2009).

[15] W.K. Bae, M.K. Nam, K. Char, S. Lee, Chem. Mater. 20, 5307 (2008). 